www.jmscr.igmpublication.org

Index Copernicus Value: 79.54

ISSN (e)-2347-176x ISSN (p) 2455-0450

crossrefDOI: https://dx.doi.org/10.18535/jmscr/v7i1.199

\title{
Journal Of Medical Science And Clinical Research
}

\section{Endobronchial Tuberculosis- A Case Report}

\section{Authors \\ Dr Gopal G. Gondchar ${ }^{1}$, Dr Sonal S. Arsude ${ }^{2 *}$, Shriyansh $^{3}$, Samruddhi B. Tayade ${ }^{4}$}

Department of Respiratory Medicine, NKP Salve Institute of Medical Sciences \& RC

Lata Mangeshkar Hospital, Nagpur, Maharashtra, India

*Corresponding Author

Dr Sonal S. Arsude

\begin{abstract}
Endobronchial tuberculosis $(E B T B)$ is an infection of tracheobronchial tree with mycobacterium. Incidence of EBTB is common in young females. High index of suspicion is required for the diagnosis. Computed tomography $(C T)$ and bronchoscopy along with microbiological investigations are useful diagnostic tools for diagnostic confirmation and for evaluation of bronchial stenosis. Early endobronchial spread of disease is better delineated by high resolution computed tomography (HRCT). The main goals for the treatment of EBTB are eradication of tubercle bacilli and prevention of bronchial stenosis. Antituberculous chemotherapy is effective in controlling the infection, but does not prevent residual bronchostenosis. Timely diagnosis and treatment with ATT along with steroids prevent stenosis in EBTB.
\end{abstract}

Keywords: Endobronchial tuberculosis, bronchoscopy, corticosteroids.

\section{Introduction}

Tuberculosis (TB) is a global challenge, with high incidence and mortality rate. ${ }^{1}$ Worldwide, around 10.0 million people developed tuberculosis in 2017 : 5.8 million were men, 3.2 million were women and 1 million were children. ${ }^{2}$ Endobronchial tuberculosis (EBTB) is infection of tracheobronchial tree along with both microbial as well as histopathological evidence of tuberculous infection. ${ }^{3}$ It is a sequelae of pulmonary tuberculosis (PTB) extending to endobronchial or endotracheal wall causing inflammation, edema, ulceration, granulation and fibrosis of the mucosa. ${ }^{4}$ Central EBTB involving proximal to lobar bronchi is more common compared EBTB involving segmental bronchi. ${ }^{5}$ Incidence of EBTB is common in young patients specially in females with common symptoms like cough with expectoration, hemoptysis, low grade fever, breathlessness and wheeze. ${ }^{1,6}$ Respiratory symptoms in EBTB are commonly nonspecific and confusing. A barking cough not responding to commonly used antitussive medication is the most common presentation slowly progressing over the period of weeks to months. ${ }^{5}$

Despite available diagnostic tests, EBTB causes morbidity as it frequently heals with concentric scarring resulting in bronchostenosis and atelectasis. Sputum examination is of prime importance towards the diagnosis of EBTB. However, in cases where sputum is negative or chest radiographic findings are equivocal, bronchoscopy along with biopsy and lavage sample and computed tomography are the investigations of choice for confirmation of diagnosis. ${ }^{1,6}$ True incidence and complications of EBTB are unidentified as bronchoscopy is not performed routinely in each PTB case. ${ }^{7}$ 


\section{Case Report}

A 49 years old female patient labour worker by occupation came to outpatient department with chief complaints of breathlessness since 2 months, fever with chills with evening rise in temperature since 45 days, dry cough since 15 days, genaralised weakness, loss of appetite and loss of weight.

On Respiratory system examination, patient had wheeze. Chest x-ray PA view was suggestive of consolidation in right lower and upper zone. Montoux test was $20 \mathrm{~mm}$ after 48 hours. On sputum examination, no organism was seen on acid fast bacilli (AFB) staining \& culture sensitivity was negative. Patient was started on broad spectrum antibiotics. After 5 days course of antibiotics, patient did not show any improvement in the symptoms, hence, decision of bronchoscopy was taken.

In bronchoscopy, Right upper lobe, middle lobe \&lower lobe showed actively caseating odematatous hyperemic lesion. Mucopurulent secretions were obtained and bronchoalveolar lavage (BAL) was sent for CBNAAT. Bronchial biopsy was suggestive of chronic granulomatous inflammation. On HRCT chest, patchy areas of consolidation in right lower lobe and patches of consolidation in right upper lobe were seen suggestive of pulmonary kochs with its sequele. CBNAAT report was suggestive of mycobacterium tuberculosis (MTB) and rifampicin resistance was not detected. Hence patient was started on antitubercular treatment (ATT) CAT 1 fixed dose combination (FDC) \& oral prednisolone $1 \mathrm{mg} / \mathrm{kg}$ for 4 weeks followed by gradual tapering of the dose. Patient was followed up after 2 months and 4 months with recheck bronchoscopy. Follow up bronchoscopy done after 4 months was suggestive of partial resolution of TB and stenosis and patient was improved in terms of signs and symptoms.

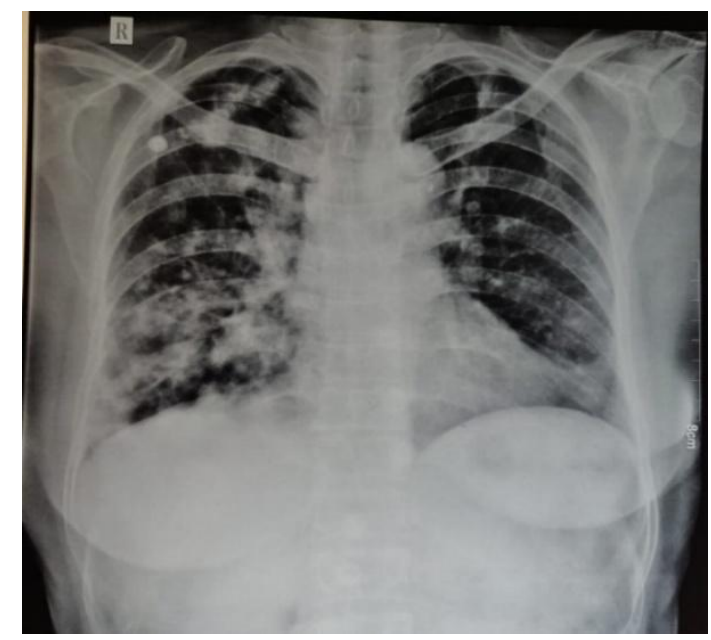

Figure 1: Chest X-ray PA view

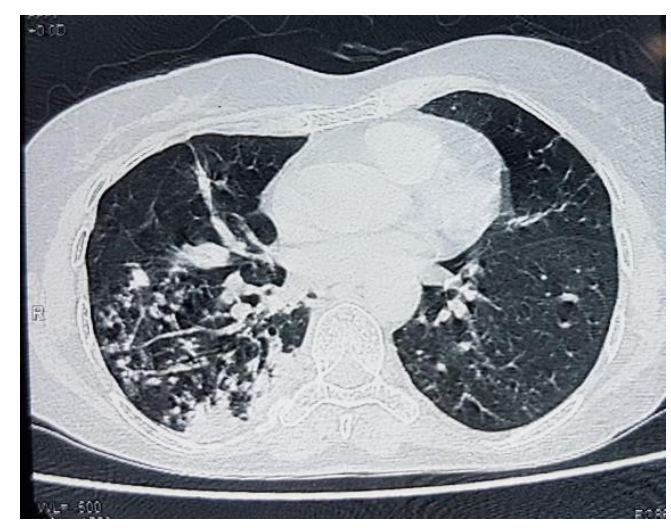

Figure 2: HRCT Thorax

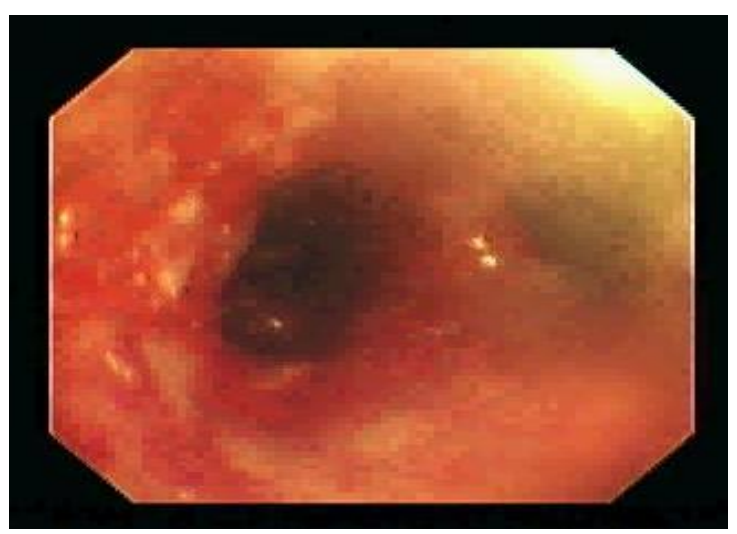

Figure 3: Bronchoscopy image showing caseation and stenosis

\section{Discussion}

WHO had declared TB as a global emergency. ${ }^{8}$ EBTB is defined as tuberculous infection in tracheobronchial tree. ${ }^{6}$ According to previous studies, EBTB was reported in $10-40 \%$ of patients with sputum positive PTB. ${ }^{8}$ Most common sites involved are right upper lobe and right main bronchus. The chest X-ray shows either 
consolidation or loss of volume. But, a normal chest X-ray does not exclude endobronchial pathology. ${ }^{6}$ Fibreoptic bronchoscopy provides excellent means for diagnosis of suspected cases of pulmonary TB especially when sputum smears are negative for AFB. ${ }^{9}$ Early endobronchial spread of disease is better delineated by high resolution computed tomography (HRCT) than a conventional chest CT. Various studies had reported a diagnostic sensitivity of $95 \%$ to $97 \%$ in endobronchial tuberculosis using HRCT. ${ }^{8}$

Our case of EBTB was a 49 years old female patient with chief complaint of dry cough with wheezing. According to literature, patients of EBTB are most commonly reported to present with cough and wheezing. ${ }^{7}$ Although EBTB is common in second or third decade, second peak has been reported in old age. ${ }^{8}$ The mechanism in elderly is likely due to diminished immune response, reactivation by exogenous MTB. ${ }^{4}$

On sputum examination, AFB was negative for organism, which is supported by Shahzad et al ${ }^{4}$ who reported that positivity of sputum AFB smear in EBTB is variable and a negative sputum smear does not exclude the diagnosis of EBTB. Endobronchial TB is classified into seven subtypes depending upon bronchoscopic appearance, namely, actively caseating, edematous-hyperemic, fibrostenotic, tumorous, granular, ulcerative and nonspecific bronchitis. ${ }^{6}$ Our case fulfilled the bronchoscopic appearance of actively caseating odematatous hyperemic lesion. BAL fluid can be subjected to molecular diagnostic methods like PCR and CBNAAT. $^{8}$ In our case, BAL was sent for CBNAAT which was positive for MTB.

The most important goal in the treatment of active EBTB is eradication of tubercle bacilli and another goal is to prevent bronchial stenosis. Beneficial effects of corticosteroids are due to antiinflammatory property which reduce local pressure due to inflammation in TB. ${ }^{4}$ In our case, we used both ATT and oral corticosteroid for 6 weeks which is justified with study by Shim $\mathrm{YS}^{10}$ who recommended steroid therapy for active caseating, oedematous hyperaemic and tumour type EBTB, as these types are prone to bronchial stenosis. Also, Roy et $\mathrm{al}^{9}$ reported that category I ATT and oral prednisolone is effective for treatment of EBTB. Three months post treatment, repeat bronchoscopy revealed restored patency of left upper lobe bronchus with stenosis.

\section{Conclusion}

The most important component for the diagnosis of EBTB is a high index of suspicion and early bronchoscopy. Timely diagnosis and treatment with ATT along with steroids prevent stenosis in EBTB. Antituberculous chemotherapy is effective in controlling the infection, but does not prevent residual bronchostenosis. We report a case of EBTB to create awareness and to avoid misdiagnosis as we believe that early detection and prompt treatment can prevent complications of EBTB.

\section{References}

1. Arwadi AM, Khalid F, Sadiq S, et al. Endobronchial tuberculosis in an immunocompetent young filipino male-A case report from Kingdom of Saudi Arabia. Surg Case Rep Rev.2018; 2(3): 1-3

2. World Health Organization. Global tuberculosis report 2018. World Health Organization; 2018

3. Agarwal P, Jagpal S, Scardella AT. Endobronchial Tuberculosis Presenting as Chronic Middle Lobe Atelectasis. American Journal of Respiratory and Critical Care Medicine. 2018; 197:A6945.

4. Shahzad T, Irfan M. Endobronchial tuberculosis - a review. Journal of thoracic disease. 2016 Dec;8(12):3797-802.

5. Kashyap S, Solanki A. Challenges in endobronchial tuberculosis: from diagnosis to management. Pulmonary medicine. 2014;2014.

6. Aneja A, Krishnaswamy UM, Thyagaraj V, et al. Endobronchial tuberculosis: two case reports and review of the literature. Case reports in pulmonology. 2014;2014. 
7. Faisal M, Harun H, Hassan TM, et al. Treatment of multiple-level tracheobronchial stenosis secondary to endobronchial tuberculosis using bronchoscopic balloon dilatation with topical mitomycin-C. BMC pulmonary medicine. 2016 Dec;16(1):53.

8. Lakshmipriya S, Sunny A, Shivashankaran S et al. Ulcerative Form of Endobronchial Tuberculosis: A Rare Case. EC Pulmonology and Respiratory Medicine. 2018;7:280-4.

9. Roy PP, Dey SK, Sarkar A, et al. Diagnosis of three cases of endobronchial tuberculosis presenting as unresolved pneumonia, following fiberopticbronchoscopic biopsy. Lung India. $2010 \mathrm{Jul} ; 27(3): 185-8$.

10. Shim YS. Endobronchial tuberculosis. Respirology. 1996 Jun;1(2):95-106. 